\title{
Medical dissolution therapy for kidney stone with low density on non- contrast computed tomography.
}

\author{
Eray Hasirci $^{\text {1*, Mehmet Ilteris Tekin }}{ }^{1}$, Ayhan Dirim ${ }^{1}$, Mehmet Resit Goren ${ }^{2}$, Mustafa Agah Tekindal ${ }^{3}$, \\ Hakan Ozkardes ${ }^{1}$ \\ ${ }^{1}$ Department of Urology, Baskent University Faculty of Medicine, Ankara, Turkey \\ ${ }^{2}$ Department of Urology, Baskent University School of Medicine, Adana Dr. Turgut Noyan Medical and Research \\ Center, Adana, Turkey \\ ${ }^{3}$ Department of Biostatistics and Medical Informatics, Izmir University Faculty of Medicine, Izmir, Turkey
}

\begin{abstract}
Objective: To review the outcome of dissolution therapy in low-density urinary stones defined by nonenhanced computed tomography.

Materials and methods: The outcome of dissolution therapy in patients treated between May 2011 and July 2016 was retrospectively reviewed. Potassium sodium hydrogen citrate was used in cases with syone of $<\mathbf{8 0 0}$ Hounsfield units determined by non-enhanced computed tomography. A decrease of $50 \%$ in the long axis of the stone was defined as partial dissolution. Cases with complete and partial stone dissolution were taken as the treatment success group whereas those who could not tolerate the treatment and who has less than $\mathbf{5 0 \%}$ decrease in stone size were noted as treatment failure. The patients were compared with respect to age, body mass index, stone size, stone density, duration of treatment and follow-up, urine $\mathrm{pH}$ and serum uric acid levels.

Results: Of 46 patients 31 completed the treatment course. A full response was obtained in $22(71 \%)$ and a partial response in $4(\mathbf{1 2 . 9 \%})$ cases. The basic factors found to affect the success of treatment were stone surface area, pre-treatment urine $\mathrm{pH}$ and serum uric acid levels.

Conclusion: Low-density urinary stones can be successfully treated with dissolution therapy. In patients with radiolucent stones, the stone density should be measured by using non-enhanced computed tomograms. In cases examined with suitable stone density, dissolution treatment can be started without determining the exact type of the stone.
\end{abstract}

Keywords: Dissolution, Medical treatment, Radiolucent stones, Urinary calculous disease.

Accepted on November 2, 2017

\section{Introduction}

It is estimated that uric acid stones constitute 8 to $10 \%$ of all renal stones [1]. The most important factors in the formation of uric acid stones are acidic urine, dehydration and hyperuricosuria because of low urine volume. Changes in the urine $\mathrm{pH}$ are the most important mechanisms in the formation of uric acid stones. Medical problems such as chronic diarrhoea, myeloproliferative diseases, insulin resistance, diabetes mellitus, and monogenic metabolic diseases such as Lesh-Nyhan syndrome, which cause hyperuricosuria, constitute a high risk for uric acid stone formation. Except in cases of severe obstruction, progressive azotemia, severe infection or persistent pain, the primary treatment for uric acid stones is dissolution therapy with urinary alkalinisation $[2,3]$. The success of empirical dissolution treatment in mixed stones is thought to be related to the reduction in the uric acid composition of the stone. The ideal start for dissolution treatment should be after analysis of stones retrieved either with spontaneous passage or endoscopic removal. This sequence however is not always possible.

Previous studies have reported that information about the stone composition can be obtained from density measurements of urinary stones on non-enhanced Computed Tomography (CT). Several in vitro studies have shown that uric acid stones can be differentiated from Calcium Oxalate $(\mathrm{CaOx})$ stones and even subtypes of calcium stones can be differentiated on CT images [4-6]. A previous in vitro study in our clinic showed that the densities of pure or mixed uric acid stones ranged between 427 and 436 Hounsfield Units (HU) on CT and the densities of calcium phosphate, calcium oxalate monohydrate, and calcium oxalate dihydrate stones were $>1000 \mathrm{HU}$ [7]. Moreover, it has been stated in two different studies that the success of shockwave lithotripsy and percutaneous nephrolithotomy can be predicted according to the Hounsfied density $[8,9]$. 
The aim of this study was to evaluate the outcome and factors that may have an impact on the outcome of dissolution therapy in low density urinary stones as measured on non-enhanced CT.

\section{Materials and Methods}

\section{Study design and population}

A retrospective examination was collected from the data of 1231 patients who were applied with non-contrast CT because of urinary stones between May 2011 and July 2016. Of these patients, urinary stones were determined in 1073, and of these, low-density renal pelvis stones were determined in 46 patients. Patients with low-density ureteral stones were excluded from the study. The medical records were reviewed of 46 patients (26 females, 20 males) who were applied with medical dissolution therapy between May 2011 and July 2016. All patients had an initial evaluation with routine blood chemistry and urinalysis, direct urinary radiogram and non-enhanced CT.

Potassium sodium hydrogen citrate treatment was administered based on low-density stone definition. A total of 10 patients who were receiving treatment such as allopurinol or thiazide, which can affect the urine volume and uric acid metabolism, were excluded from the study. All patients had pre-treatment records of Body Mass Index (BMI), stone surface area, stone density on CT, spot urine $\mathrm{pH}$ and serum uric acid level. Stones were located in the renal pelvis in all cases. Before starting treatment, a JJ stent was placed in 6 patients because of obstruction and persistent pain. In 2 cases with solitary kidney, nephrostomy drainage was required because of deteriorated renal function, and pyonephrosis was drained with nephrostomy in 1 case.

The initial dose of treatment was $2.5 \mathrm{~g}$ tid. After the $4^{\text {th }} \mathrm{d}$ of treatment, the patients were requested to take urinary $\mathrm{pH}$ measurements with $\mathrm{pH}$ strips before each dose for 2 days. The patients reported their $\mathrm{pH}$ measurements on the $7^{\text {th }} \mathrm{d}$. Through these measurements, dose adjustments were made in order to keep the urinary $\mathrm{pH}$ in the range of 6.5-6.8.

The outcome of treatment was evaluated in a follow-up visit after a mean period of 3 months (range; 2-6 months). Complete disappearance of the stone on follow-up CT was defined as full response and a reduction of at least $50 \%$ in stone size was defined as partial response. The CT images of a patient at pretreatment and with full response at the end of treatment are shown in Figures 1 and 2. Patients with complete and partial responses were regarded as the treatment success group whereas the remaining cases and cases non-compliant to treatment were regarded as the unsuccessful group.

\section{CT protocol}

The tomograms were obtained in a four-detector helical CT scanner (Sensation, Siemens, Germany). The scans were obtained with $1 \mathrm{~mm}$ slice thickness and at two energy levels of 80 and $120 \mathrm{kV}$. The current was kept constant at $240 \mathrm{~mA}$. All the CT images were evaluated by a single radiologist (senior radiology resident). All the CT scans were assessed in the axial, coronal and sagittal planes. The number, dimensions, location and CT density (in Hounsfield Unit) of the stones were recorded for each patient. The density measurements were made after bone filtering as these were the sharpest images with greater spatial resolution. Three different $0.01 \mathrm{~cm}^{2}$ Region-of-Interest (ROI) measurements in Hounsfield units were obtained for each stone. The mean of the 3 measurements was accepted as the absolute $\mathrm{CT}$ value for a particular stone (Figure 3). The densities of the uric acid stones were found to be $311 \mathrm{HU}$ (range 112-436 HU), $587 \pm 329 \mathrm{HU}$, and $541.5 \pm$ 161.1 HU in three different studies [7,10,11]. Stones with density $<800 \mathrm{HU}$ were accepted as low density stones with a uric acid component and treatment was planned accordingly.

Stone surface area was calculated using the formula, Stone surface area $=$ maximum diameter $\times$ width $\times \pi \times 0.25$ [12]. In patients with multiple calculi, the total surface area of the stones was taken into consideration.

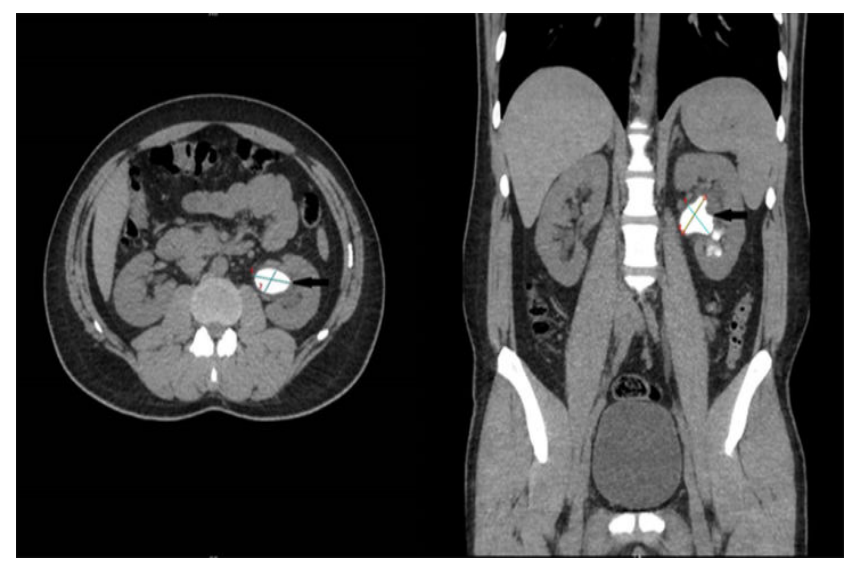

Figure 1. Image of a stone $37 \times 35 \times 21 \mathrm{~mm}$ in size of $350 \mathrm{HU}$ density in the left kidney in the renal pelvis. Pre-treatment axial and vertical image. The arrow indicates a renal pelvis stone.

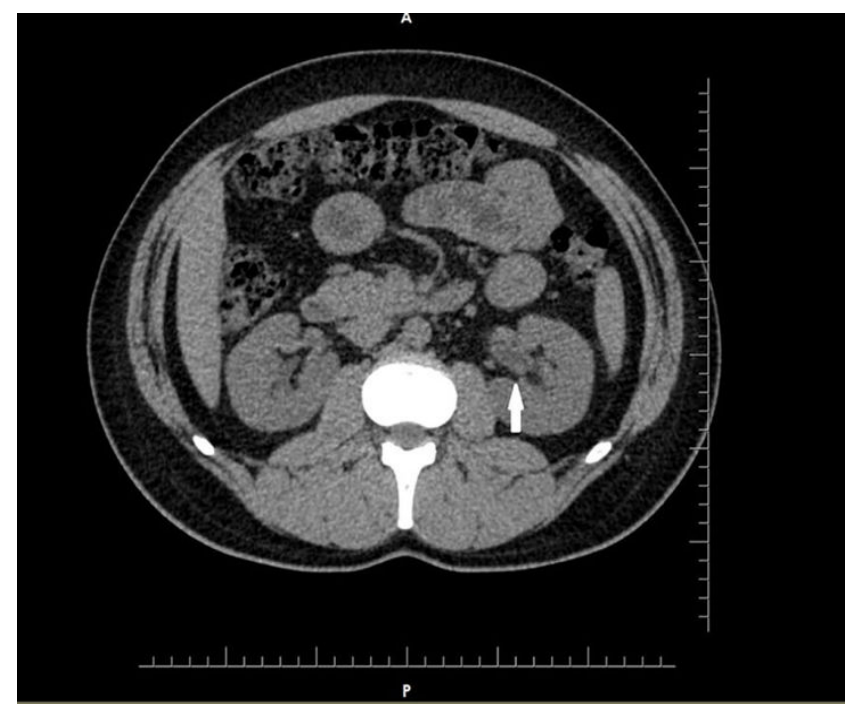

Figure 2. Image taken at the 7-month follow-up examination of a stone in the left kidney in the renal pelvis with full response obtained after 5 months of treatment. The arrow indicates a renal pelvis with no stone. 


\section{Statistical analysis}

Data obtained in the study were analysed using SPSS v.17 software (SPSS Inc., Chicago, IL, USA). For categorical and continuous variables, descriptive statistics were given (mean, Standard Deviation (SD), median, minimum, maximum, number (n) and percentage (\%)). The homogeneity of variance from pre-conditions of parametric tests was tested with the Levene test. The normality hypothesis was examined with the Shapiro Wilks test. To evaluate the differences between two groups, the Student's t-test was used when the pre-conditions of parametric tests were met and the Mann Whitney U-test when they were not. A value of $p<0.05$ was accepted as statistically significant.

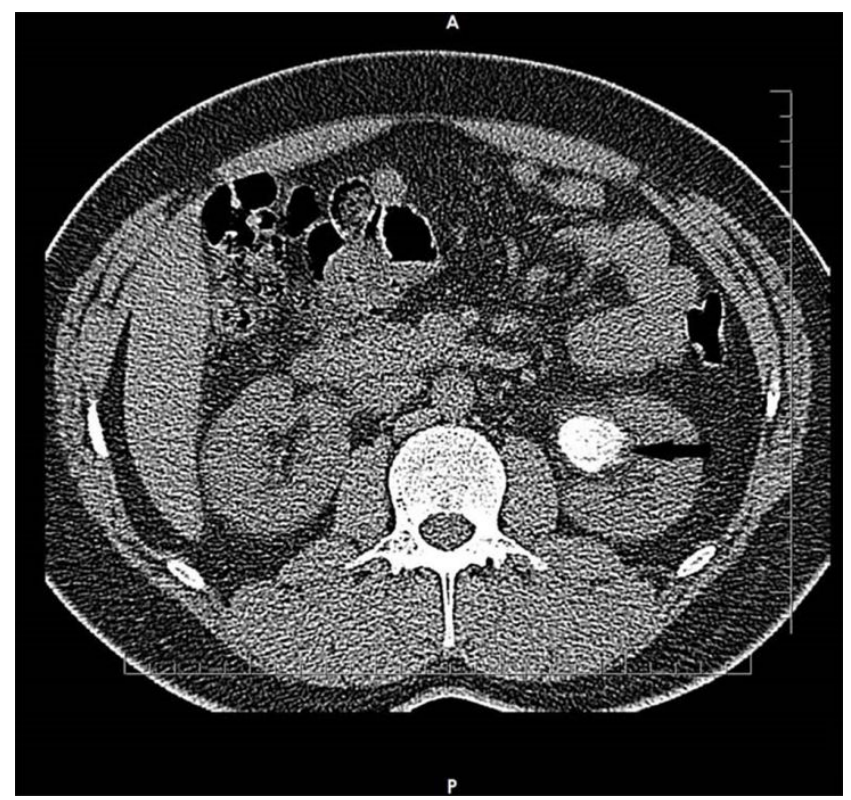

Figure 3. Image of the non-contrast CT density measurement of the stone. The arrow indicates a renal pelvis stone. The density of the stone is $350 \mathrm{HU}$.

\section{Results}

Urinary alkalinisation treatment was recommended to a total of 36 patients with radiolucent and low density $(<800 \mathrm{HU})$ stones. Of these, 5 patients did not attend follow-up examinations or rejected treatment. The remaining 31 patients (35\% male) had a mean age of 63.3 years (range; 44-82 y).

Full response was obtained in 22 patients $(71 \%)$ and there were 4 cases $(12.9 \%)$ with a partial response. The treatment failed in 5 patients $(16.1 \%)$. All the patients $(n=6)$ with pure uric acid stones verified by stone analysis before treatment were in the successful group. The successful and unsuccessful groups were compared in respect of mean age (62.6 vs. 66.2 years, $\mathrm{p}=0.163), \mathrm{BMI}(\mathrm{p}=0.519)$ and stone density (578.4 vs. $594 \mathrm{HU}$, $\mathrm{p}=0.786)$ (Table 1).

The pre-treatment parameters determined to be significantly different between the groups were stone surface area, spot urine $\mathrm{pH}$ and serum uric acid levels. The mean stone surface area was $2.55 \mathrm{~cm}^{2}$ in the successful group and $5.09 \mathrm{~cm}^{2}$ in the unsuccessful group $(\mathrm{p}<0.05)$. The mean urine $\mathrm{pH}$ was 5.17 in the successful group and 5.5 in the unsuccessful group $(\mathrm{p}<0.05)$. The mean serum uric acid levels were determined as $6.4 \mathrm{mg} / \mathrm{dL}$ in the successful group and $8.92 \mathrm{mg} / \mathrm{dL}$ in the unsuccessful group $(\mathrm{p}<0.01)$.

In addition to density determination on the CT images, sufficient volume for analysis of the stones was obtained in only 6 cases. X-ray diffraction analysis revealed pure uric acid stones in all 6 cases.

Table 1. Factors affecting the success of dissolution treatment.

\begin{tabular}{|c|c|c|c|}
\hline & \multicolumn{2}{|l|}{ Dissolution } & \multirow[t]{2}{*}{$\mathbf{p}$} \\
\hline & Successful & Unsuccessful & \\
\hline Age & $62.6 \pm 11.3$ & $66.2 \pm 2.28$ & 0.163 \\
\hline $\mathrm{BMI}\left(\mathrm{kg} / \mathrm{cm}^{2}\right)$ & $29.38 \pm 4.51$ & $30.28 \pm 2.3$ & 0.519 \\
\hline Stone area $\left(\mathrm{cm}^{2}\right)$ & $2.55 \pm 1.95$ & $5.09 \pm 6.2$ & 0.046 \\
\hline Density (HU) & $578.4 \pm 122.7$ & $594 \pm 65.8$ & 0.786 \\
\hline Treatment duration (w) & $17.58 \pm 14.49$ & $30 \pm 23.58$ & 0.124 \\
\hline Follow-up duration (w) & $34.77 \pm 31.29$ & $39.6 \pm 30.6$ & 0.753 \\
\hline Pre-treatment Urine $\mathrm{pH}$ & $5.17 \pm 0.28$ & $5.5 \pm 0.5$ & 0.045 \\
\hline $\begin{array}{l}\text { Pre-treatment Serum uric acid level } \\
(\mathrm{mg} / \mathrm{dl})\end{array}$ & $6.4 \pm 1.55$ & $8.92 \pm 0.84$ & 0.001 \\
\hline
\end{tabular}

(BMI: Body Mass Index; cm: Centimetre, dl: Decilitre, HU: Hounsfield Unit, Kg: Kilogram, mg: milligram).

\section{Discussion}

There is an increasing prevalence of urinary stone disease, but in parallel, the widespread use of non-contrast CT has facilitated the diagnosis of stone diseases [13]. Due to the high resolution and high sensitivity of non-contrast CT in current use, this has replaced 'excretory urography' in the diagnosis of stone disease. However, although non-contrast CT can determine the stone location, size, number and density, it does not provide effective information about the stone composition.

In the current treatment of stone disease, treatment methods used by many urologists are minimally invasive approaches such as percutaneous nephrolithotomy, flexible or semi-rigid ureterorenoscopy and shock-wave lithotripsy. Unfortunately, there has not yet been a standardisation of indications and treatment. The ability of the surgeon, and facilities and preferences of technique can affect the decision made. In addition, the composition of the stones and location, size and number play an important role in the selection of treatment. The determination of the composition of the stone before treatment can be of great benefit for patients, especially for those to be treated medically, such as with urinary alkalisation.

In previous studies it has been attempted to determine the stone types by combining radiological and urinary parameters without direct analysis of the spontaneously passed or surgically removed stones [14-16]. Torricelli et al. developed a nomogram based on age, BMI and $24 \mathrm{~h}$ urine to differentiate 
Calcium Oxalate $(\mathrm{CaOx})$ and uric acid stones [17]. However, differentiation of $\mathrm{CaOx}$ and uric acid stones in that study could only be made when the predominant component was more than $50 \%$. It was concluded that this had a negative impact on the chance of full dissolution. In prediction of the response of urinary stones to treatment, non-enhanced CT HU density measurements are extremely valuable. Previous studies have shown that $\mathrm{CaOx}$ stones can be differentiated from uric acid, struvite and cystine stones with HU measurements on noncontrast CT $[5,18]$. In a study using ex vivo analysis it was demonstrated that dual-source CT technology was able to differentiate hydroxyapatite stones from $\mathrm{CaOx}$ stones or uric acid stones from $\mathrm{CaOx}$ stones [19].

Non-contrast CT is accepted as the gold standard in the diagnosis of urinary system stone disease. However, studies have reported that because of the high dose applied in this technique and as it does not provide data about the composition of the stone, it is insufficient. Alsyouf et al. examined the effect of the results of non-contrast CT applied with a low dose (5-140 mAs) and with a conventional dose on HU measurements and stone diagnosis and no significant correlation was determined of the reduced dose with HU [20]. In a review by Bres-Niewada et al. it was reported that despite high-dose application of non-contrast CT, insufficient data were provided in the diagnosis of stone disease [21].

With the emergence of Dual-Energy CT (DECT) as a good option, studies in recent years have shown the DECT can be used in the sub-grouping of calcium stones and for in vivo classification of urinary stones. This technique is even recommended for the determination of calcium oxalate monohydrate stones that are resistant to lithotripsy [22]. In another study which examined the efficacy of DECT in the classification of urinary system stone disease, $78.6 \%$ accuracy was determined in calcium stones and it was therefore reported to have high sensitivity in this respect [23].

The main point is the identification of uric acid stones when dissolved with oral chemolysis. In a study by Deveci et al., pure uric acid stones were shown to be mean $311 \mathrm{HU}$ (range, 112-436 HU) and mixed uric acid stones were mean $331 \mathrm{HU}$ (range, 110-427 HU) [7]. In another study, the mean density of uric acid stones was reported as mean 541.5 $\pm 161.1 \mathrm{HU}$ [11]. Although there is no standard value for standard density of uric acid stones on non-contrast CT, in the light of the previous research it seems to be $<700 \mathrm{HU}$. In the current study, the threshold value was accepted as $800 \mathrm{HU}$ with the consideration that stones could be pure or with a uric acid component. Accordingly, the administration of medical dissolution treatment was found to be appropriate.

In a previous study with a limited number of patients, alkalinisation was applied to patients with radiolucent stones and acidic urine with elevated uric acid output in $24 \mathrm{~h}$ urine. At the end of a $12 \mathrm{w}$ treatment period, complete response was obtained in 3 of 8 patients [3]. In the current study, potassium sodium hydrogen citrate was used for alkalinisation. Consistent with the study by Torricelli et al. the dissolution therapy was found to be successful at a high rate in patients with pure uric acid stones [17]. In a study where patients were grouped as pure uric acid stones or uric acid component stones, the groups differed with regard to serum uric acid levels [24]. While the serum uric acid levels of patients with pure uric acid stones were found to be higher, the levels decreased significantly relative to a gradual reduction of the uric acid component of the stone. When the results of these two studies are evaluated together, the serum uric acid levels and the success of treatment are expected to be low in patients with a low uric acid component stone [17,24]. The results of those studies deviate from those of the current study. The serum uric acid levels of the unsuccessful group of the current study were found to be significantly higher $(p<0.01)$. Reichard et al. showed lower urinary $\mathrm{pH}$ measurements in cases of pure uric acid stones. This finding is consistent with the current study data since the urinary $\mathrm{pH}$ levels were found to be lower in the successful group $(\mathrm{p}<0.05)$ [24].

Stewart et al. investigated the accuracy of determining the type of stone using non-enhanced CT density measurements. The accuracy of density measurements was investigated on stones in the density range of 700 to $1,000 \mathrm{HU}$, thus those which were predicted to be basically calcium oxalate stones. The overall accuracy of prediction was $32 \%$. While the prediction rate in small stones $(<5 \mathrm{~mm})$ was $100 \%$, it decreased to $41 \%$ in medium-size $(5-10 \mathrm{~mm})$ and $45 \%$ in large stones $(>10 \mathrm{~mm})$. That study emphasized that there was a greater presence of mixed components in larger stones, which in turn made accurate prediction more difficult [25]. The stone areas that appeared to have an impact on the accuracy of stone type prediction and thus the institution of empirical dissolution therapy were significantly different in the successful and unsuccessful treatment groups in the present study. This finding warrants further investigation with larger groups of cases. However, the addition of allopurinol to medical treatment may be considered when the stone burden is greater.

Previous studies have shown only a $10 \%$ success rate for dissolution therapy when treatment was commenced based solely on radiolucency on direct radiograms [26]. It has been suggested that the most important reason for this treatment failure is the mixed composition of the stones and a calcium covering layer which prevents contact of the uric acid crystals with the alkalinised urine [27]. The density measurement technique in the present study took an average of three sites of measurement and thus revealed $>800 \mathrm{HU}$ density for the stones with multiple components including a harder outer shell. This led to a meticulous selection of cases for dissolution therapy, consequently increasing the success rate.

\section{Limitations}

This study had several limitations. First, this study was retrospective. Secondly, there was a bias in the patient selection. In our institution, as suggested by the American Urological Association and European Association of Urology, the criteria are followed of therapeutic procedures, including medication, shockwave lithotripsy, ureterorenoscopy and percutaneous nephrolithotomy. Even if stones were large, some 
patients were resistant to dissolution therapy and patients may want a definitive result in a short time. The third limitation is that the urine parameters were examined on spot urine. Some tests such as magnesium and citrate in the $24 \mathrm{~h}$ urine test could have been of guidance. Finally, as the patient numbers of those who received dissolution therapy and those in the unsuccessful group were low compared to the other group, this could have affected the results. Therefore, there is a need for further, better designed, prospective, randomised studies of high case volume which could contribute to the treatment algorithm.

\section{Conclusion}

Urinary alkalinisation treatment can be administered based on the stone density measurements taken on non-enhanced CT and in the absence of stone analysis. Improvements in CT technology are promising for increasing the accuracy of stone type prediction, which in turn forms a robust basis for starting dissolution therapy in selected cases that do not have retrieved stones and thus stone analysis before treatment.

\section{Compliance with Ethical Standards}

\section{Conflict of interest}

There was no funding associated with this study. The authors have no conflict of interests to declare.

\section{Ethical approval}

All procedures performed in studies involving human participants were in accordance with the ethical standards of the institutional review board (Baskent University Institutional Review Board (Project no: KA16/365)).

\section{References}

1. Sakhaee K. Epidemiology and clinical pathophysiology of uric acid kidney stones. J Nephrol 2014; 27: 241-245.

2. Cicerello E, Merlo F, Maccatrozzo L. Urinary alkalization for the treatment of uric acid nephrolithiasis. Arch Ital Urol Androl 2010; 82: 145-148.

3. Trinchieri A, Esposito N, Castelnuovo C. Dissolution of radiolucent renal stones by oral alkalinization with potassium citrate/potassium bicarbonate. Arch Ital Urol Androl 2009; 81: 188-191.

4. Kuwahara M, Kageyama S, Kurosu S, Orikasa S. Computed tomography and composition of renal calculi. Urol Res 1984; 12: 111-113.

5. Nakada SY, Hoff DG, Attai S, Heisey D, Blankenbaker D, Pozniak M. Determination of stone composition by noncontrast spiral computed tomography in the clinical setting. Urology 2000; 55: 816-819.

6. Patel SR, Haleblian G, Zabbo A, Pareek G. Hounsfield units on computed tomography predict calcium stone subtype composition. Urol Int 2009; 83: 175-180.

7. Deveci S, Coskun M, Tekin MI, Peskircioglu L, Tarhan NC, Ozkardes H. Spiral computed tomography: role in determination of chemical compositions of pure and mixed urinary stones an in vitro study. Urology 2004; 64: 237-240.

8. Ouzaid I, Al-qahtani S, Dominique S, Hupertan V, Fernandez P, Hermieu JF, Delmas V, Ravery V. A 970 Hounsfield units (HU) threshold of kidney stone density on non-contrast computed tomography (NCCT) improves patients' selection for extracorporeal shockwave lithotripsy (ESWL): evidence from a prospective study. BJU Int 2012; 110: 438-442.

9. Gucuk A, Uyeturk U, Ozturk U, Kemahli E, Yildiz M, Metin A. Does the Hounsfield unit value determined by computed tomography predict the outcome of percutaneous nephrolithotomy? J Endourol 2012; 26: 792-796.

10. Narayan VM, Bozorgmehri S, Ellen JH, Canales MT, Canales BK, Bird VG. Evaluating region of interest measurement strategies to characterize upper urinary tract stones on computerized tomography. J Urol 2017; 197: 715-722.

11. Kawahara T, Miyamoto H, Ito H, Terao H, Kakizoe M, Kato Y, Ishiguro H, Uemura $\mathrm{H}$, Yao M, Matsuzaki J. Predicting the mineral composition of ureteral stone using non-contrast computed tomography. Urolithiasis 2016; 44: 231-239.

12. Ather MH, Nazim SM, Sulaiman MN. Efficacy of semirigid ureteroscopy with pneumatic lithotripsy for ureteral stone surface area of greater than $30 \mathrm{~mm} 2$. J Endourol 2009; 23: 619-622.

13. De SK, Liu X, Monga M. Changing trends in the American diet and the rising prevalence of kidney stones. Urology 2014; 84: 1030-1033.

14. Kulkarni NM, Eisner BH, Pinho DF, Joshi MC, Kambadakone AR, Sahani DV. Determination of renal stone composition in phantom and patients using singlesource dual-energy computed tomography. J Comput Assist Tomogr 2013; 37: 37-45.

15. Motley G, Dalrymple N, Keesling C, Fischer J, Harmon W. Hounsfield unit density in the determination of urinary stone composition. Urology 2001; 58: 170-173.

16. Spettel S, Shah P, Sekhar K, Herr A, White MD. Using Hounsfield unit measurement and urine parameters to predict uric acid stones. Urology 2013; 82: 22-26.

17. Torricelli FC, De S, Liu X, Calle J, Gebreselassie S, Monga M. Can 24-hour urine stone risk profiles predict urinary stone composition? J Endourol 2014; 28: 735-738.

18. Altan M, Citamak B, Bozaci AC, Gunes A, Dogan HS, Haliloglu M, Tekgul S. Predicting the stone composition of children preoperatively by Hounsfield unit detection on non-contrast computed tomography. J Pediatr Urol 2017; 13: 505 .

19. Matlaga BR, Kawamoto S, Fishman E. Dual source computed tomography: a novel technique to determine stone composition. Urology 2008; 72: 1164-1168.

20. Alsyouf M, Smith DL, Olgin G, Heldt JP, Lightfoot M, Li $\mathrm{R}$, Baldwin DD. Comparing stone attenuation in low- and 
conventional-dose noncontrast computed tomography. J Endourol 2014; 28: 704-707.

21. Bres-Niewada E, Dybowski B, Radziszewski P. Predicting stone composition before treatment-can it really drive clinical decisions? Cent European J Urol 2014; 67: 392-396.

22. Acharya S, Goyal A, Bhalla AS, Sharma R, Seth A, Gupta AK. In vivo characterization of urinary calculi on dualenergy CT: going a step ahead with sub-differentiation of calcium stones. Acta Radiol 2015; 56: 881-889.

23. Chaytor RJ, Rajbabu K, Jones PA, McKnight L. Determining the composition of urinary tract calculi using stone-targeted dual-energy CT: evaluation of a low-dose scanning protocol in a clinical environment. Br J Radiol 2016; 89: 20160408.

24. Reichard C, Gill BC, Sarkissian C, De S, Monga M. 100\% uric acid stone formers: what makes them different? Urology 2015; 85: 296-298.

25. Stewart G, Johnson L, Ganesh H, Davenport D, Smelser W, Crispen P, Venkatesh R. Stone size limits the use of
Hounsfield units for prediction of calcium oxalate stone composition. Urology 2015; 85: 292-295.

26. Sinha M, Prabhu K, Venkatesh P, Krishnamoorthy V. Results of urinary dissolution therapy for radiolucent calculi. Int Braz J Urol 2013; 39: 103-107.

27. Kaneko K, Yoshida N, Okazaki K, Yamanobe T, Yamaoka N, Yasuda M, Ogata N, Yamada Y, Uchida S, Fujimori S. Urinary stone analysis in a patient with hyperuricemia to determine the mechanism of stone formation. Nucleosides Nucleotides Nucleic Acids 2011; 30: 1072-1076.

\section{*Correspondence to}

Eray Hasirci

Department of Urology

Baskent University Faculty of Medicine

Turkey 